\title{
The risk factors and preventive measures for the recurrence of endometrial polyps
}

\author{
Nitisha Doobaly ${ }^{1}$, Xiuli Wang ${ }^{2 *}$, Minjuan Lin ${ }^{1}$, Shu Zhu², Xia Wang ${ }^{1}$
}

\begin{abstract}
${ }^{1}$ Department of Obstetrics and Gynecology, ${ }^{2}$ Department of Gynecology, The First Affiliated Hospital of Nanjing
\end{abstract} Medical University, Nanjing, Jiangsu Province, China

Received: 14 January 2019

Accepted: 19 January 2019

*Correspondence:

Dr. Xiuli Wang,

E-mail: xiuli_2266@163.com

Copyright: (c) the author(s), publisher and licensee Medip Academy. This is an open-access article distributed under the terms of the Creative Commons Attribution Non-Commercial License, which permits unrestricted non-commercial use, distribution, and reproduction in any medium, provided the original work is properly cited.

\begin{abstract}
Endometrial polyps (EPs) are a frequently encountered gynecologic disease with abnormal uterine bleeding and infertility being the two common presenting problems, and hysteroscopic polypectomy is an effective method to remove them. The postoperative polyp recurrence might result in reappearance of abnormal uterine bleeding or infertility, whereas factors influencing the postoperative recurrence potential have limited data. Endometrial polyp recurrence remains a concern with recurrence rates of $2.5 \%$ to $43.6 \%$. As such, it is critical to identify the risk factors and the preventive measures for endometrial recurrence, especially in reproductive-age women desiring future conception, to aid in clinical counselling and decision making. The recurrence of EPs is related to estrogen stimulation and endometrial hyperplasia. The progesterone-containing drugs are currently the most commonly used method to prevent the recurrence of EPs. In this article, authors aim to discuss the high-risk factors of EPs recurrence and the preventive measures for EPs recurrence. The preventive measures will focus on the combined oral contraceptives (COCs) and the levonorgestrel-releasing intrauterine system (LNG-IUS).
\end{abstract}

Keywords: Contraceptives, Endometrial polyps, Hysteroscopy, Recurrence

\section{INTRODUCTION}

EPs are benign localized overgrowth of endometrial tissue, composed of glands, stroma, and blood vessels covered by epithelium. ${ }^{1}$ It is a common gynaecologic condition, with an incidence of up to $24 \%-41 \%$ in women with abnormal uterine bleeding and $10 \%$ of asymptomatic women, relying on the population studied. ${ }^{2}$ Abnormal uterine bleeding is the most common complaint of symptomatic patients. EPs are single in most of the patients although in $12-20 \%$ of the cases those are multiple. ${ }^{3}$ In a variable proportion of cases $(0-$ $12.9 \%$ ) depending on the population studied can develop malignant transformation.,4 EPs might also result in infertility by intracavitary bleeding or presenting an abnormal environment for embryo implantation, and the presence of EPs is suggested to be the most common pathologic finding detected by office hysteroscopy in subjects with recurrent implantation failure..$^{5,6}$

EPs can be diagnosed by ultrasound, sono-hysterography, hysteroscopy and curettage. Among them, hysteroscopy is superior to the other three methods due to the fact that it is able to detect the number, type, and location of EPs. Hysteroscopic polypectomy is now the criterion standard for the diagnosis and treatment of EPs. It is performed under direct visualization to completely remove the polyps with adjacent endometrium left intact. ${ }^{7}$ Hysteroscopic polypectomy appears to enhance fertility and increase pregnancy rates for infertile women with no 
other cause to explain their infertility. ${ }^{5,8}$ However, although hysteroscopic resection effectively removes EPs, studies demonstrated the postoperative recurrence rates of EPs to range from $2.5 \%$ to $43.6 \%$, depending on the follow-up duration and nature of polyps. ${ }^{7-10}$ Therefore, it is important to attempt to identify risk factors associated with recurrence and to take measures to prevent recurrence of EPs.

\section{HIGH-RISK FACTORS OF EPS RECURRENCE}

\section{Clinical factors and polyp number}

Concerning potential predictive clinical risk factors such as age, parity, BMI, history of hypertension or diabetes mellitus, tamoxifen treatment, HRT and PCOS status do not have an influence on endometrial polyp recurrences. ${ }^{7}$ Studies have shown that body mass index do not contribute to higher endometrial polyp recurrence rates despite the notion that higher levels of circulating estrogen secondary to obesity contribute to abnormal endometrial stimulation. However, the age, hypertension and menopausal status are indicated as risk factors of malignant polyps. ${ }^{1}$ Recurrence can occur very soon after surgery, and the incidences are much more frequent in patients with high number of EP. Studies demonstrated that a high number of EPs increase the risk of recurrence in premenopausal women, with a recurrence rate of $45.5 \%$ compared with $13.4 \%$ in the single endometrial patients after one year follow up. ${ }^{11}$ EPs number $>6$ is associated with a much higher recurrence than a single EP, although endometrial polyp numbers 2-3 have similar recurrence to single endometrial polyp. ${ }^{12}$ In addition, it is reported that a high number of EPs are associated with an increased postoperative recurrence potential of polyps at the same location. ${ }^{9}$ Resectoscopic surgery allows the complete removal of polyps leaving intact the adjacent endometrium. In this context, a recurrence should be considered as a 'new' polyp, although it is difficult to determine retrospectively if recurrence develops in a location near or far from the previous polyp. ${ }^{7}$ However, 'blind' methods, as simple curettage, can fail in extracting the entire polyp also in $60-87 \%$ of the cases and lead to a fragmentation and facilitated its reconstitution, which cannot be considered as a real recurrence. $^{7,13}$

\section{Histopathologic features}

Histopathologic features such as benign polyps, hyperplastic polyps without atypia and cancerous polyps play an important role in the recurrence of EPs. The histopathologic type represents an independent predictive risk factor in the recurrence. Indeed, the risk of recurrence is significantly higher in hyperplastic polyp without atypia compared with benign polyps, while less than $10 \%$ of benign one did the same. ${ }^{7}$ There is also more frequent progression of the hyperplastic polyps without atypia towards forms of symptomatic recurrences or precancerous or cancerous forms that require more radical surgery, such as hysterectomy. However, even benign polyps, and among them also those that are asymptomatic, have a certain ability to recur, and therefore it is recommenced that these also are removed. ${ }^{4}$

The type of EPs, either pedunculated or sessile, does not play a role in the postoperative recurrence potential. The two types of EPs, pedunculated and sessile are determined based on the angle that is made between the polyp and the adjacent wall. Pedunculated type polyps being defined with the angle of polyp surface to endometrium $<90$ degrees, while sessile polyps are those with an angle $>90$ degrees. ${ }^{9}$ However, the pedunculated polyps are strongly associated with the presentation of abnormal uterine bleeding. In comparison with sessile polyps, the surface of pedunculated EPs is at risk of touching the endometrium of the opposite uterine wall, and abnormal uterine bleeding occurs consequently. ${ }^{9}$

In addition, follow-up duration plays an important role in the recurrence of polyps. The recurrence of potential of EPs increases as the follow-up duration is longer. This positive association between the recurrence rate and follow-up period is also found in endometriosis, in which the 2-year recurrence rate is $21.5 \%$ postoperatively, whereas the 5-year recurrence rate is as high as $40-50 \% .^{9}$

\section{Resection methods of endometrial polyps}

Hysteroscopic surgery is the first-line therapeutic option in the treatment of patients with EPs. It can achieve complete resection of EPs, with an overall complication rate of $0.38 \%$, however, studies have shown that hysteroscopic resection is associated with a recurrence of $2.5 \%$ to $3.7 \% .^{8,14}$ And studies have illustrated a high endometrial polyp recurrence rate of $13.3 \%$ on a mean follow-up of 2 years in women treated with resectoscopic hysteroscopy. $^{7}$ In comparison with hysteroscopic resection, intrauterine morcellation is associated with lower recurrence of EPs. ${ }^{15}$ Intrauterine morcellation, using a nonelectrical energy-dependent mechanical device, allows deeper resection of tissue down to the base of the endometrial polyp than using micro scissors or polyp-grasping forceps does. Also, recurrence of EPs is one of the most common problems in breast cancer patients receiving long-term treatment with tamoxifen, with a recurrence rate of $7.7 \%$ in post-menopausal patients. ${ }^{16}$ Each year of additional use of tamoxifen is estimated to increase the risk of developing recurrent polyps by up to five-fold. Patients treated with tamoxifen that underwent polypectomy associated with endometrial resection had an endometrial recurrence rate of $8.54 \% .{ }^{10}$ To prevent recurrence of this condition, a simultaneous endometrial polypectomy with endometrial resection should be performed. Polypectomy with endometrial ablation could reduce abnormal vaginal bleeding and pathological sonographic findings, which therefore would reduce the need for or completely avoid the need for invasive procedures, such as hysteroscopy or curettage. Thus, the need for the use of anesthetic and surgical 
resources would not be necessary. Therefore, for postmenopausal breast cancer patients, suffering from EPs associated with tamoxifen, hysteroscopic polypectomy with endometrial ablation is considered as a safe and effective treatment method, preventing the recurrence of EPs. ${ }^{10}$

\section{PREVENTIVE MEASURES FOR RECURRENCE OF EPS}

\section{Recurrence of EPs is related to estrogen stimulation and endometrial hyperplasia}

Over the course of the menstrual cycle, human endometrium undergoes repeated proliferation and apoptosis, and the alteration of this proliferationapoptosis balance may result in EP formation or hyperplasia. ${ }^{17}$ EPs are usually soft, pedunculated, or sessile tissue growths consisting of variable amounts of endometrial glands, stromal tissue, and vessels, which can result in irregular bleeding or spotting, heavy periods, and fertility problems. The frequency of EPs in the general population ranges from $6 \%$ to $38 \%$, with peak incidence between 40 and 50 years of age. ${ }^{3,18}$ EPs may arise in response to unopposed estrogen, as well as other growth stimuli, including insulin and insulin-like and epithelial growth factors that can also cause endometrial hyperplasia. Continuous exposure to estrogen unopposed by progesterone, polycystic ovary syndrome, and tamoxifen use are well-recognized risk factors for benign endometrial hyperplasia (BEH). Hormone replacement therapy (with or without progesterone) and tamoxifen treatment have been associated with the development of EP, presumably mediated through agonist estrogenic effects. ${ }^{19-21}$ The occurrence of EPs is also closely related to estrogen stimulation and endometrial hyperplasia.

Recurrence of EP after surgical removal has been demonstrated to occur in $15 \%$ to $60 \%$ different hormonal approaches have been used as therapy for EPs, such as progestin and gonadotropin-releasing hormone agonist (GnRHa), combined oral contraceptives (COCs) and the levonorgestrel-releasing intrauterine system (LNGIUS). ${ }^{8,14}$ These hormonal approaches can also be used as the measures to prevent the recurrence of EPs. It is reported that GnRHa gives a short-term symptomatic relief for EPs. ${ }^{22}$ Progestin with high anti-estrogenic activity is most effective at preventing development of EP when compared to different hormone replacement regimes in EP formation. ${ }^{23}$ Hence, the progesteronecontaining drugs are currently the most commonly used method to prevent the recurrence of EPs. We will focus on COCs and LNG-IUS to prevent the recurrence of EPs.

\section{COCs}

COCs combine an estrogen and a progestin in a single tablet that is ingested orally once daily in a variety of dosing regimens ranging from conventional 21 active and 7 hormone-free tablets to reduced hormone-free interval regimens (e.g. 24/4) to regimens with some days of lowdose estrogen-only tablets in lieu of placebo tablets to continuous-use regimens. Most COCs employ ethinyl estradiol for the estrogen component, although estradiolbased pill regimens have been recently introduced. In contradistinction to the few estrogenic compounds available in COCs, there is a wide array of contraceptive progestins available in COCs, with most of the older progestins being derived from testosterone, new compounds not derived from testosterone like nomegestrol acetate and dienogest and other novel progestins such as drospirenone being derived from spirolactone.

COCs have been shown to control the growth of the endometrium, decreasing the risk of both endometrial hyperplasia and EPs. However, the response of polyps to oral contraceptive is different depending on the shape of polyp. Sessile polyps are more sensitive to oral contraceptives treatment than pedunculated polyp that is sessile polyps regress in a higher rate than pedunculated polyps under oral contraceptive treatment. ${ }^{24}$

\section{$L N G-I U S$}

The LNG-IUS is a safe and highly effective long-lasting reversible contraceptive. While shorter-acting contraceptives such as the oral contraceptive pill require high levels of patient compliance to maintain effectiveness, the LNG-IUS is effective for up to 5 years, which a first-year failure rate similar to that of sterilization $(0.2 \%){ }^{25}$ The LNG-IUS contains a total of levonorgestrel $52 \mathrm{~g}$ with a release rate of $20 \mu \mathrm{g} /$ day for 5 years, after which the daily dose drops by approximately $50 \% .{ }^{26}$ It is cost-effective and suitable for the vast majority of women. ${ }^{25,27}$ The LNG-IUS can also be used to treat endometrial hyperplasia and EPs. ${ }^{28,29}$ Moreover, the endometrial progestin concentration observed in women treated with the LNG-IUS is shown to be one hundredfold higher compared to that in the endometrial mucosa after oral therapy. ${ }^{30}$ According to a meta-analysis, the LNG-IUS is superior to oral progestin therapy in the treatment of endometrial hyperplasia. LNG-IUS offers convenience, minimal adverse effects, reversibility, and long-term endometrial protection. There is a fair quality of evidence indicating that the use of the LNG-IUS is safe for women with endometrial hyperplasia and may have therapeutic benefits. ${ }^{31}$

Oral tamoxifen is a standard adjuvant therapy for women with breast cancer. It has been shown to improve diseasefree and overall survival in women with hormone receptor positive disease and the usual treatment period is 5 years. ${ }^{32}$ However, it exerts weak estrogenic effect on the endometrium and hence is associated with various endometrial pathologies. The incidences of EPs, hyperplasia and endometrial cancer had been reported to be between 5 and $35 \%, 4.7-16 \%$ and $0.8-5 \%$, respectively. ${ }^{33-35}$ The use of LNG-IUS among female users of tamoxifen has shown a significant reduction in 
the incidence of EPs. ${ }^{36-38}$ Wong et al conducted a RCT with 129 women with breast cancer and who were users of tamoxifen; the women were randomised to LNG-IUS before initiation of tamoxifen intake or no treatment. The uterine cavity was evaluated at 12, 24, 45 and 60 months after baseline. No cases of endometrial hyperplasia were recorded in either groups; however, LNG-IUS reduced EP de novo. ${ }^{39}$ Additionally, Arnes et al evaluated 59 women with hyperplastic polyps; the women received an LNG-IUS, 10mg of oral MPA (10 days per cycle) or only observation for 6 months. The numbers of women without polyps after 6 months were 18 of 18 for those who received an LNG-IUS, 5 of 20 for women treated with cyclic MPA (25\%) and 2 of 21 (9\%) for those without any treatment. ${ }^{40}$

\section{CONCLUSION}

The number of endometrial polyps and follow-up duration, but not the type of polyps, are major factors that determine the recurrence potential after hysteroscopic polypectomy. A higher number of endometrial polyps and longer follow-up duration are associated with a greater potential of polyp recurrence. Nevertheless, the correlation between recurrence and hyperplastic appearance of the polyp cannot be underestimated, and therefore we must emphasize the importance and the need for further validation by prospective and multicentre long-term follow-up studies. If considering recurrence rate is high enough, it is recommendable to remove all verified EPs, benign ones and hyperplastic ones with atypia as they have an even higher propensity to recur. Furthermore, hormonal approaches can also be used as measures to prevent endometrial polyp recurrence.

\section{ACKNOWLEDGMENTS}

This work was supported by the second phase of maternal and child health key talent project of Jiangsu Province (FRC201709), the first phase of maternal and child health project of Jiangsu Province (F201619), the fifth phase of the "333 project" of Jiangsu Province (LGY2016003).

\section{Funding: No funding sources}

Conflict of interest: None declared

Ethical approval: Not required

\section{REFERENCES}

1. Savelli L, De Iaco P, Santini D, Rosati F, Ghi T, Pignotti E, et al. Histopathologic features and risk factors for benignity, hyperplasia, and cancer in EPs. Am J Obstet Gynecol. 2003;188:927-31.

2. DeWaay DJ, Syrop CH, Nygaard IE, Davis WA, Van Voorhis BJ. Natural history of uterine polyps and leiomyomata. Obstet Gynecol. 2002;100:3-7.

3. Orovieto R, Bar-Hava I, Dicker D, Bar J, Ben-Rafael Z, Nezi A. EPs during menopause: characterization and significance. Acta Obstet Gynecol Scand. 1999;78:883-6.
4. Golan A, Cohen-Sahar B, Keidar R, Confrea A, Ginath S, Sagiv R. EPs: symptomatology, menopausal status and malignancy. Gynecol Obstet Invest. 2010;70:107-12.

5. Varasteh NN, Neuwirth RS, Levin B, Keltz MD. Pregnancy rates after hysteroscopic polypectomy and myomectomy in infertile women. Obstet Gynecol. 1999;94:168-71.

6. Hosseini MA, Ebrahimi N, Mahdavi A, Aleyasin A, Safdarian L, Fallahi P, et al. Hysteroscopy in patients with repeated implantation failure improves the outcome of assisted reproductive technology in fresh and frozen cycles. J Obstet Gynaecol Res. 2014;40:1324-30.

7. Paradisi R, Rossi S, Scifo MC, Dall'O'F, Battaglia C, Venturoli S. Recurrence of EPs. Gynecol Obstet Invest. 2014;78:26-32.

8. Preutthipan S, Herabutya Y. Hysteroscopic polypectomy in 240 premenopausal and postmenopausal women. Fertil Steril. 2005;83:705-9.

9. Yang JH, Chen CD, Chen SU, Yang YS, Chen MJ. Factors influencing the recurrence potential of benign EPs after hysteroscopic polypectomy. PLoS One. 2015;10:e0144857.

10. Gao W, Zhang L, Li W, Li J, Wang W, Zhao W, et al. Three-year follow-up results of polypectomy with endometrial ablation in the management of EPs with tamoxifen in Chinese women. Eur J Ob-stet Gynecol Reprod Biol. 2012;161:62-5.

11. Gu F, Zhang H, Ruan S, Li J, Liu X, Xu Y, et al. High number of endometrial polyps is a strong predictor of recurrence: findings of a prospective cohort study in reproductive-aged women. Fertil Steril. 2018;109:493-500.

12. Taylor E, Gomel V. The uterus and fertility. Fertil Steril. 2008;89:1-16.

13. Ebauer G, Hafner A, Siebzehnrübl E, Lang N. Role of hysteroscopy in detection and extraction of EPs: results of a prospective study. Am J Obstet Gynecol. 2001:184:59-63.

14. Henriquez DD, vanDongen $H$, Wolterbeek R, Jansen FN. Polypectomy in premenopausal women with abnormal uterine bleeding: effectiveness of hysteroscopic removal. J Minim Invasive Gynecol. 2007;14:59-63.

15. AlHilli MM, Nixon KE, Hopkins MR, Weaver AL, Laughlin-Tommaso SK, Famuyide AO. Long-term outcomes after intrauterine morcellation vs. hysteroscopic resection of endometrial polyps. J Minim Invasive Gynecol. 2013;20:215-21.

16. Biron-Shental T, Tepper R, Fisheman A, Shapira J, Cohen I. Recurrent endometrial polyps in postmenopausal breast cancer patients on tamoxifen. Gynecol Oncol. 2003;90(2):382-6.

17. Banas T, Basta P, Knafel A, Skotniczny K, Jach R, Hajdyla-Banas I, et al. DFF45 expression in human endometrium is associated with menstrual cycle phases and decreases after menopause. Gynecol Obstet Invest. 2012;73:177-82. 
18. Torrijos MC, de Merlo GG, Mirasol EG, García MT, Parra CÁ, Goy EI. Endometrial study in patients with postmenopausal metrorrhagia. Arch Med Sci. 2016;12:597-602.

19. Maja JH, Barbosa IC, Marques D, Calmon LC, Ladipo LC, Coutinho EM. Hysteroscopy and transvaginal sonography in menopausal women receiving hormone replacement therapy. J Am Assoc Gynecol Laparose. 1996;4:1318.

20. Cheng W-f, Lin H-H, Trong P-L, Huang S-C. Comparison of endometrial changes among symptomatic tamoxifen-treated and non-treated premenopausal and postmenopausal breast cancer patients. Gynaecol Oncol. 1997;66:233-7.

21. Reslova T, Tonsor J, Resi M, Kugler R, Vavrova I. Endometrial polyps-A clinical study of 245 cases. Arch Gynecol Obstet. 1999;262:3Z.

22. Vercellini P, Trespidi L, Bramante T, Panazza S, Mauro F, Crosignani PG. Gonadotropin-releasing hormone agonist treatment before hysteroscopic endometrial resection. Int $\mathbf{J}$ Gynaecol Obstet. 1994;45:235-9.

23. Oguz S, Sargin A, Kelekci S, Aytan H, Tapisiz OL, Mollamahmutoglu L. The role of hormone replacement therapy in endometrial polyp formation. Maturitas. 2005;50:231-6.

24. Wada-Hiraike O, Osuga Y, Hiroi H, Fujimoto A, Maruyama M, Yano T, et al. Sessile polyps and pedunculated polyps respond differently to oral contraceptives. Gynecol Endocrinol. 2011;27(5):3515.

25. Stoddard A, McNicholas C, Peipert JF. Efficacy and safety of long-acting reversible contraception. Drugs. 2011;71(8):969-80.

26. Sivin I, Stern J, Coutinho E, Mattos CE, El Mahgoub S, Diaz S, et al. Prolonged intrauterine contraception: a seven-year randomized study of the levonorgestrel $20 \mathrm{mcg} /$ day ( $\mathrm{LNg} \mathrm{20}$ ) and the copper T380 Ag IUDS. Contraception. 1991;44(5):473-80.

27. National Institute for Health and Care Excellence. Long-acting reversible contraception. In: National Institute for health and care excellence, ed. Clinical guidelines; 2014.

28. American College of Obstetricians and Gynecologists. Noncontraceptive uses of hormonal contraceptives. ACOG Practice Bulletin No. 110. Obstet Gynecol. 2010;115(1):206-18.

29. Bahamondes L, Ribeiro-Huguet P, Andrade KC, Leon-Martins O, Petta CA. Levonorgestrel-releasing intrauterine system (Mirena) as a therapy for endometrial hyperplasia and carcinoma. Acta Obstet Gynecol Scand. 2003;82(6):580-2.

30. Barrington JW, Bowen-Simpkins P. The levonorgestrel intrauterine system in the management of menorrhagia. Br J Obstet Gynaecol. 1997;104:614-6.

31. Whiteman MK, Zapata LB, Tepper NK, Marchbanks PA, Curtis KM. Use of contraceptive method among women with endometrial hyperplasia: a systematic review. Contracept. 2010;82:56-63.

32. Early Breast Cancer Trialists' Collaborative Group. Effects of chemotherapy and hormonal therapy for early breast cancer on recurrence and 15-year survival: an overview of the randomised trials. Lancet. 2005;365:1687-717.

33. Neven P, De Muylder X, Van Belle Y, Van-Hooff I, Vanderick G. Longitudinal hysteroscopic follow-up during tamoxifen treatment. Lancet. 1998;351:36.

34. Vosse M, Renard F, Coibion M, Neven P, Nogaret JM, Hertens D. Endometrial disorders in 406 breast cancer patients on tamoxifen: the case for less intensive monitoring. Eur J Obstet Gynecol Reprod Biol. 2002;101:58-63.

35. Ozsemer S, Ozaran A, Itil I, Dikmen Y. Endometrial pathology of 104 postmenopausal breast cancer patients treated with tamoxifen. Eur J Gynaecol Oncol. 1998;19:580-3.

36. Chin J, Konje JC, Hickey M. Levonorgestrel intrauterine system for endometrial protection in women with breast cancer on adjuvant tamoxifen. Cochrane Database Syst Rev. 2009;4:CD007245.

37. Gardner FJ, Konje JC, Abrams KR, Brown LJ, Khanna S, AL-Azzawi, et al. Endometrial protection from tamoxifen-stimulated changes by a levonorgestrel-releasing intrauterine system: a randomised controlled trial. Lancet. 2000;356:17117.

38. Gardner FJ, Konje JC, Bell SC, Abrams KR, Brown LJ, Taylor DJ, et al. Prevention of tamoxifen induced endometrial polyps using a levonorgestrel releasing intrauterine system long-term follow-up of a randomised control trial. Gynecol Oncol. 2009;114:452-6.

39. Wong AW, Chan SS, Yeo W, Yu MY, Tam WH. Prophylactic use of levonorgestrel-releasing intrauterine system in women with breast cancer treated with tamoxifen: a randomized controlled trial. Obstet Gynecol. 2013;121:943-50.

40. Arnes M, Hvingel B, Orbo A. Levonorgestrelimpregnated intrauterine device reduces occurrence of hyperplastic polyps: a population-based follow-up cohort study. Anticancer Res. 2014;34:2319-24.

Cite this article as: Doobaly N, Wang X, Lin M, Zhu S, Wang X. The risk factors and preventive measures for the recurrence of endometrial polyps. Int J Reprod Contracept Obstet Gynecol 2019;8:78791. 